Dokuz Eylül Üniversitesi-Mühendislik Fakültesi

Fen ve Mühendislik Dergisi

Cilt 20, Sayı 58, Ocak, 2018
Dokuz Eylul University-Faculty of Engineering Journal of Science and Engineering Volume 20, Issue 58, January, 2018

DOI: $10.21205 /$ deufmd. 2018205808

\title{
A Survey of BSDF Measurements and Representations
}

\author{
Murat KURT*1 \\ ${ }^{1}$ International Computer Institute, Ege University, 35100, Izmir. \\ (ORCID: 0000-0002-3236-5595)
}

(Alınıs / Received: 06.02.2017, Kabul / Accepted: 29.11.2017, Online Yayınlanma / Published Online: 20.01.2018)

\begin{abstract}
Keywords
Appearance model,

Appearance capture, Bidirectional scattering distribution function, BSDF, Rendering.

Abstract: Measuring and representing light reflection and transmission accurately are core to high fidelity visual simulation of materials. In this paper, we analyze state-of-the-art Bidirectional Scattering Distribution Function (BSDF) measurements and models. We show that the most of the state-ofthe-art BSDF models do not suggest a general solution for any surface class, from glasses to metals, isotropic to anisotropic materials, and daylight redirecting films. Furthermore, it's shown that an accurate and dense BSDF acquisition is not a trivial task at especially some specific measurement angles, such as normal incidence and grazing angles. In this paper, we address the problem of finding a general solution for efficient BSDF measurement and representation. We also outline the main issues that do not allow the effective use of current BSDF representations. Finally, we suggest open research issues that need to be investigated in BSDF literature.
\end{abstract}

\section{BSDF Ölçümlemelerinin ve Sunumlarının İncelenmesi}

\begin{abstract}
Anahtar Kelimeler Özet: Işık yansıması ve iletiminin doğru bir şekilde ölçümlenip Görünüm modeli, Görünüm ölçümlemesi, İki yönlü saçılım dağılım fonksiyonu, BSDF, Görüntüleme. sunulması malzemelerin yüksek doğrulukta görsel simülasyonu için çok önemlidir. Bu makalede, literatürdeki en son İki Yönlü Saçılım Dağllım Fonksiyonu (BSDF) ölçümlemeleri ve modelleri incelenmektedir. Literatürdeki en son BSDF modellerinin camlardan metallere, izotropikden anizotropiğe ve işığı yeniden yönlendiren filmlere kadar bir çok yüzey tipi için genel bir çözüm önermedikleri gösterilmiştir. Bunun dışında, özellikle normal geliş yönü ve süpürme açlları gibi spesifik ölçümleme açllarında doğru ve yoğun BSDF ölçümlemesinin kolay olmadığı gösterilmiştir. Bu makalede, etkin BSDF ölçümlemesi ve sunumu için genel bir çözüm bulma problemine yönelinmiştir. Ayrıca, mevcut BSDF sunumlarının etkin olarak kullanımına müsaade etmeyen ana konular belirtilmiștir. Son olarak, BSDF literatüründe incelenmesi gereken açık araştırma konuları önerilmiştir.
\end{abstract}




\section{Introduction}

Rendering complex scenes requires precise descriptions of materials involved. Some materials, such as papers, glasses, metals and daylight redirecting films, have unique appearances. To measure the reflectance/transmittance of these materials accurately, various measurement devices are used [2-4]. These measured datasets are represented by various material models, such as Bidirectional Scattering Distribution Function (BSDF), or Bidirectional Reflectance Distribution Function (BRDF) or Bidirectional Transmittance Distribution Function (BTDF) models [5-6].

Data acquisition process often yields noisy, irregular and sparse measurements, especially when higher dimensional data needs to be measured (i.e., anisotropic measurements). Furthermore, some data acquisition systems [4] do not allow measurements at some specific measurement angles, such as normal incidence and grazing angles. This is another reason for sparsely measured data. Therefore, representing sparse, irregular and noisy measurements with a material model accurately is an open challenge.

Analytical BRDF models [7-17], BTDF models $[1,6,18]$ and BSDF models [1,6,18-19] try to represent measurements with a few parameters. But, analytical models fail to fit some material types (i.e., structured glasses). Data-driven based representations [2,2023] are more successful to represent real-world materials. However, datadriven based representations pose some difficulties when measurements are sparse, irregular and noisy.

In this work, we investigate state-of-theart BSDF measurements and models. We expose advantages and disadvantages of the investigated BSDF measurements and models. We also summarize the main challenges that do not permit the effective use of the most of the state-ofthe-art BSDF representations. We focus on the problem of finding a general framework for an accurate and efficient BSDF acquisition and representation. Finally, we propose open research issues that need to be investigated in BSDF literature.

\section{An Analysis of BSDF}

This section can be decomposed to the following two subdomains: an analysis of BSDF acquisition systems and an analysis of BSDF representations.

\subsection{An analysis of BSDF}

\section{measurements}

One of the most popular BRDF database is the MERL MIT database. BRDFs in the MERL MIT database have been acquired by Matusik et al. [2]. This database was constructed by using an image-based BRDF measurement setup, which can be seen in Figure 2(a). Matusik et al.'s measurement setup captures isotropic BRDF data by a series of photographs taken from a surface of a sphere. These photographs include light reflection data from various view and light orientations [24]. The MERL MIT database consists of 100 different isotropic BRDF measurements which include highly dense and regular samples. In the MERL MIT database, each material is represented by Rusinkiewicz coordinate system [25], which is known to represent highly specular peaks more effectively than the standard coordinate system. There are 1.458 .000 measurements for each material and these measurements are suitable for direct rendering. Therefore, this database has been used by many researchers [16,22-23,26-27] for validation, experimental analysis and comparison purposes.

Ngan et al. [26] measured 4 different anisotropic BRDFs by using an image 


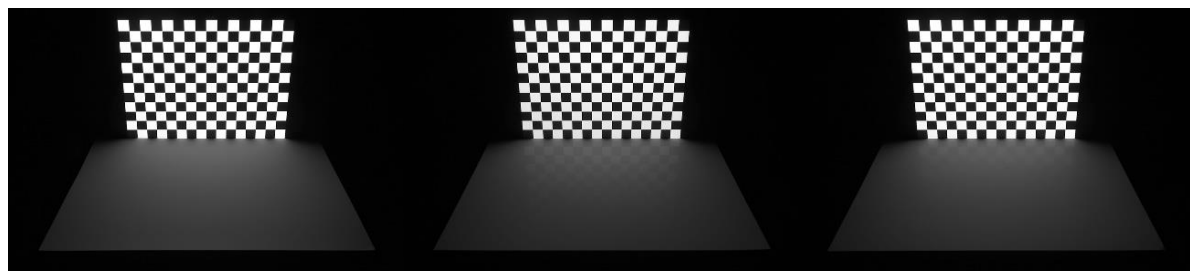

(a)

(b)

(c)

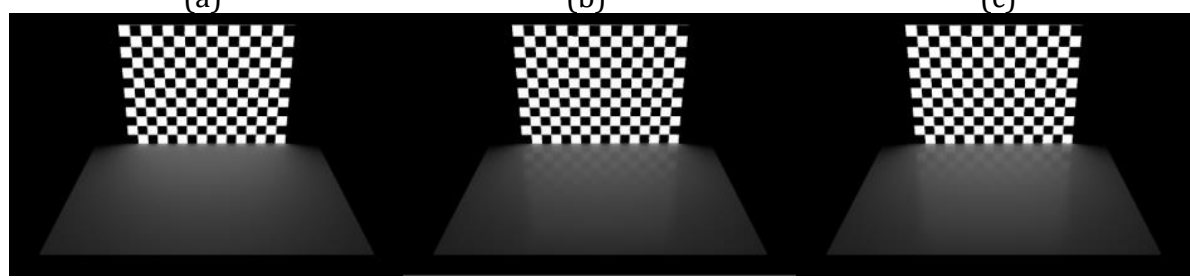

(d)

(e)

(f)

Figure 1. Isotropic translucent paper materials are measured and represented by Papas et al. [1]. (a), (d) Matte paper. (b), (e) Glossy paper. (c), (f) Luster paper. While images in the first row are real photographs, images in the second row are rendered by Papas et al.'s BSDF model (images from [1])

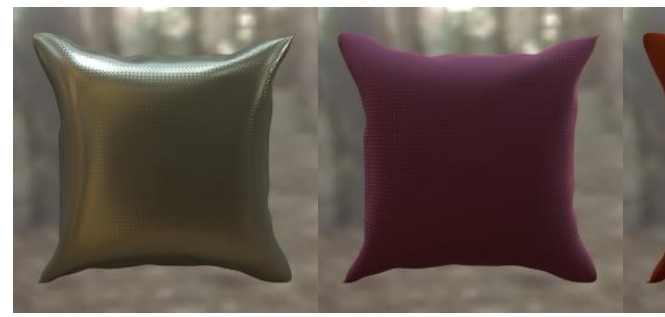

(a) (b)

$\left(L^{2}=0.0104\right)$

$\left(L^{2}=0.0006\right)$

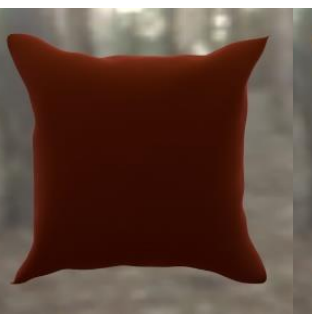

(c) $\left(L^{2}=0.0003\right)$

(d)

Figure 3. (a) Brushed aluminum, (b) Purple satin, (c) Red velvet and (d) Yellow satin materials are represented by Kurt et al.'s anisotropic BRDF model [16]. The $L^{2}$ errors of Kurt et al.'s BRDF model are also reported (images from [16])

-based BRDF acquisition setup, which is suitable for flat and flexible samples. To make anisotropic measurements, strips of materials at different orientations are wrapped around a cylinder, which can be rotated by a high precision motor. A series of photographs is taken by a fixed camera through rotating a light source and the cylinder. To form an HDR image, a set of 8 pictures with different exposures is taken for each light and target position [24]. The renderings of the measured anisotropic materials using Kurt et al.'s [16] BRDF model can be seen in Figure 3. Since anisotropic BRDF domain is four dimensional (4D) and it's very time consuming to measure whole 4D domain, this data set includes noisy, irregular and sparse measurements. Therefore, Ngan et al.'s anisotropic BRDF data set is not suitable for data-driven based representations and it must be preprocessed before it can be represented by a suitable BRDF model. In addition, both Matusik et al. [2] and Ngan et al. [26] did not address the light transmission to describe BTDFs which are required to render translucent surfaces.

Walter et al. [6] measured 4 different isotropic BTDFs from different types of rough glass surfaces, namely ground glass, etched glass, frosted glass and antiglare glass. Walter et al.'s imagebased measurement setup includes a 


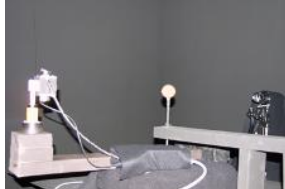

(a)

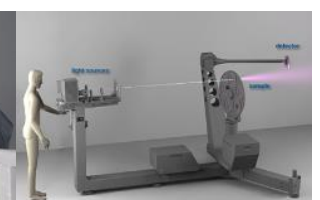

(b)
Figure 2. (a) A photograph of Matusik et al.'s BRDF measurement device [2], (b) An overview of pgII gonio-photometer [4] (images from $[2,4]$ )

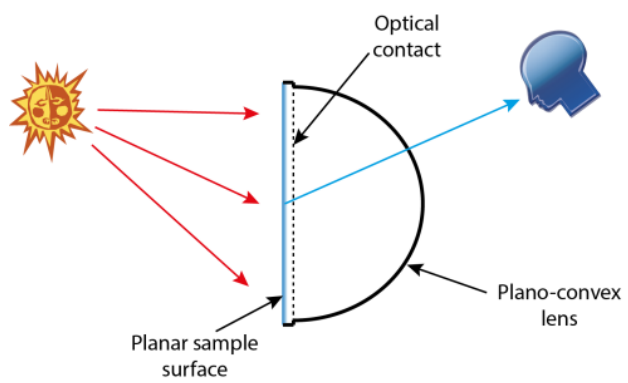

Figure 4. An overview of Walter et al.'s [6] image-based measurement setup

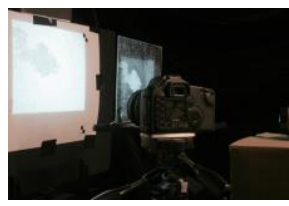

(a)

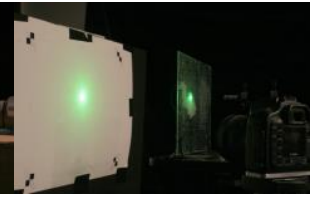

(b)
Figure 5. (a) A photograph of Gu et al.'s BSDF measurement device [28] which was used to capture the optical thickness parameter of their proposed BSDF model, (b) A photograph of Gu et al.'s BSDF measurement device [28] which was used to capture the scattering parameter of their proposed BSDF model (images from [28])

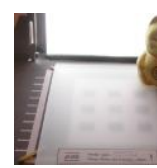

(a)

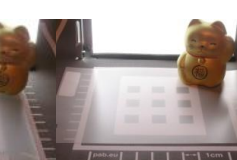

(b)

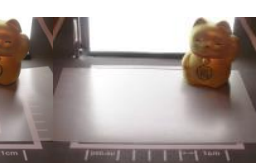

(c)
Figure 6. Photographs of isotropic translucent materials, which were measured by Apian-Bennewitz [4]. (a) L02 - 148, (b) vk_op10, (c) vk_rms220 materials (images from [4])

plano-convex lens, which is a nearly hemisphere, and it's cemented to the back of the sample (see Figure 4). This setup provides the scattered light to leave from the surface with minimal loss due to Fresnel reflection. The sample is illuminated from the rough side by a DC regulated fiber illuminator. The transmitted light is sensed from the hemispherical side by a charge coupled device (CCD) camera. The measurement is done by taking photographs and averaging the pixel values in a fixed rectangle in the image. However, this process produces measurements proportional to the BTDF times the cosine of the incident angle. Walter et al.'s goal was to validate their proposed analytical BTDF model and compare various microfacet distributions, such as Beckmann and GGX distributions. Therefore, they did not focus on making regular and non-sparse measurements. Additionally, Walter et al. [6] did not address the light reflection to describe BRDFs which are required to represent whole BSDFs of translucent materials.

$\mathrm{Gu}$ et al. [28] made some measurements from 30 different transparent materials which include contaminants, such as dust, dirt and lipids. In this image-based measurement process, $\mathrm{Gu}$ et al. [28] estimated the optical thickness and the scattering parameters of their proposed BSDF model. To measure these parameters, Gu et al. used two different measurement setups, which can be seen in Figure 5. To capture the spatially varying optical thickness $\tau(x, y)$, Gu et al. used a shadow map generated by attenuation from the contaminant layer. As it can be seen in Figure 5(a), the projector illuminates a thin transparent material which includes a contaminant layer. There are a Lambertian board behind the material and a camera on the side. The intensity of each point in the shadow map and the intensity in the clean regions of the transparent materials were used to estimate the spatially varying optical thickness parameter $\tau(x, y)$, which can be formalized as follows: 


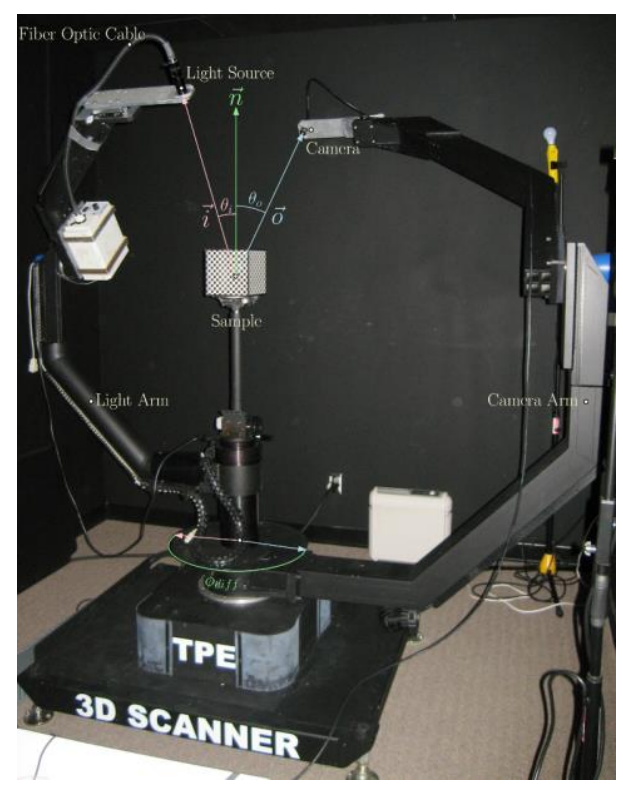

Figure 7. An overview of Papas et al.'s [1] image-based measurement setup. A checkerboard material is measured for testing purposes

$$
\tau(x, y)=-\ln \left(\frac{I(x, y)}{I_{\text {clean }}}\right),
$$

where $I(x, y)$ is the image intensity of the point $(x, y), I_{\text {clean }}$ is the image intensity in the clean regions of the measured glass. To capture the scattering parameter $g$, Gu et al. used a similar measurement setup to the previous one, except in two places (see Figure 5(b)). Firstly, they used a laser beam instead of a projector. Secondly, they used a transparent material with a uniform contaminant layer. When the laser beam hits the contaminants, it scatters towards the Lambertian board and generates a lobe pattern on the board. Gu et al. used the lobe pattern to estimate the scattering parameter $g$ of their proposed BSDF model. This estimation is based on the following formulation:

$$
\begin{aligned}
& I(x, y) \\
& =\beta p(\theta, g) \frac{e^{-\tau}-e^{-\tau / \cos \theta}}{1-\cos \theta} \cos ^{4} \theta,
\end{aligned}
$$

where $\beta$ is a scale factor, $p(\theta, g)$ is the Henyey-Greenstein phase function, $\theta$ is the angle of the scattered ray from the normal, $g$ is the scattering parameter, $\tau$ is the optical thickness parameter (see Eq. (1)), $I(x, y)$ is the average intensity in the measured lobe pattern on the board. An important advantage of Gu et al.'s measurement process is that it requires only a single image for parameter estimation.

Dai et al. [18] made 3 different Spatially Varying Bidirectional Transmission Distribution Function (SVBTDF) measurements from optically thin, transparent materials, namely grid sheet, leaf glass (See Figure 11) and rice paper. In this process, Dai et al. used a cameraCRT monitor system to capture 2D BTDF slices at each surface point. At each surface point, 2D BTDF slices are acquired by illuminating the material samples from back with CRT monitor and capturing the images from one view. A white dot on a black background on the CRT monitor is displayed at different screen positions to produce a point light source at different locations. Dai et al. [18] selected CRT monitor instead of LCD monitor, because its radiance is more uniform with changing the viewing angle. The camera's position and orientation (an angle of $\theta=45^{\circ}$ from vertical axis) are fixed, and they are calibrated before data capturing. The captured 2D BTDF slices are interpolated to a regularlysampled hemispherical function and the dual microfacet model is fitted at each surface point. In the fitting process, the weighting factor and the Microfacet Normal Distribution Function (NDF) terms of the dual-microfacet model are estimated. However, this camera-CRT monitor system and the dual-microfacet model cannot be used to represent whole BSDFs of transparent materials.

Apian-Bennewitz [4] made BRDF, BTDF and BSDF measurements by using pgII 
gonio-photometer, and he constructed an open source BME database from these measurements. Images of some measured isotropic translucent materials can be seen in Figure 6. As it can be seen in Figure 2(b), pgII gonio-photometer consists of a sample holder, a light source and a detector. A variety of sample holders can be used depending on the material. Light sources are mounted on a standard optical bench, including collimating optics and custom baffles. The detector is mounted at the end of a linkage consisting of two light weight arms, and it's moved fast at a constant distance around the sample center. While pgII gonio-photometer allows many outgoing angle measurements, it allows only a few number of incoming angle measurements. Because of this reason, measurements from BME database are irregular and sparse, and they must be preprocessed before representing and rendering measured translucent materials.

Papas et al. [1] measured 3 different isotropic BSDFs from homogeneous translucent paper materials by using an image based measurement setup, which can be seen in Figure 7. The renderings of these isotropic materials using Papas et al.'s [1] BSDF model and the real photographs of these materials can be seen in Figure 1. Papas et al. used the same parameterization $\left(0^{\circ} \leq \theta_{i}<\right.$ $90^{\circ}, 0^{\circ} \leq \theta_{o}<90^{\circ}$, and $0^{\circ} \leq \phi_{\text {diff }}<$ $180^{\circ}$ ) as Matusik et al. [2] in their measurements. Papas et al. performed full hemispherical BRDF measurements and in-plane BTDF measurements in 10 degree increments and 5 degree increments, respectively. The measured paper materials are illuminated from their front surfaces with a 150-watt quartz halogen bulb with DC regulated output. A CCD camera is positioned to front surface and back surface of paper materials for acquiring BRDFs and BTDFs, respectively. A set of 5 images are acquired and averaged to remove random noise for each shutter speed stop. The resulting image is then subtracted from a black image which visualizes a fixed pattern noise for that shutter speed. Papas et al.'s BSDF dataset is sparsely measured, and these BSDF measurements are not suitable for datadriven based BSDF representations. They must be preprocessed before they can be represented by a suitable BSDF representation.

\subsection{An analysis of BSDF models}

Noisy, irregular and sparse reflectance and transmittance measurements can be represented by analytical BRDF models [7-17], BTDF models [1,6,18] or BSDF models $[1,6,18]$. However, this kind of irregular and sparse measurements cannot be represented by any datadriven material models.

Ngan et al. [26] have experimentally validated that BRDF models, which include Fresnel term [29], can represent the measurements at grazing angles and normal incidence more accurately than its competitors. To compute Fresnel term, Ngan et al. [26] used Schlick's approximation, which is formulized as:

$$
\begin{aligned}
& F\left(\omega_{i}, \omega_{h}, f_{0 r}\right)=f_{0 r}+ \\
& \left(1-f_{0 r}\right)\left(1-\left(\omega_{i} \cdot \omega_{h}\right)\right)^{5},
\end{aligned}
$$

where $\omega_{i}$ is incoming light vector, $\omega_{o}$ is outgoing view vector, $\omega_{h}=\left(\omega_{i}+\right.$ $\left.\omega_{o}\right) /\left\|\omega_{i}+\omega_{o}\right\|$ is halfway reflection vector, $f_{0 r}$ is the Fresnel coefficient for the incident side of the surface. For example, Ashikhmin-Shirley [11], CookTorrance [7] and Kurt et al. [16] BRDF models include Fresnel term. Therefore, these BRDF models generally give better fitting results than Ward [9] and WardDuer [12] BRDF models which do not include any Fresnel terms. This result can also be seen in Figure 8. In Figure 8, the Peak Signal-to-Noise Ratio (PSNR) values [30] and difference images are 


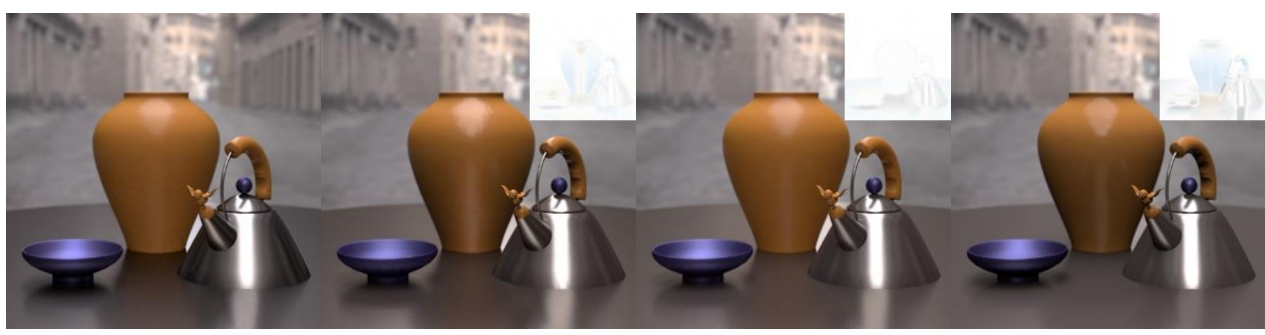

(a) Reference

(b) Ashikhmin-Shirley

(c) Cook-Torrance

(d) Edwards

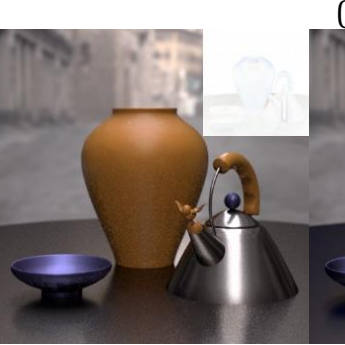

(e) Lawrence $(P S N R=31.8419)$

$(P S N R=31.1835)$

$(P S N R=26.4502)$

(f) Ward

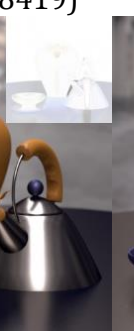

Thys

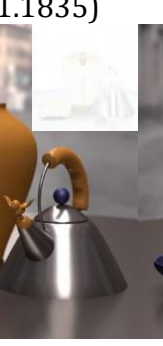

(g) Ward-Duer

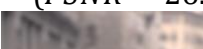

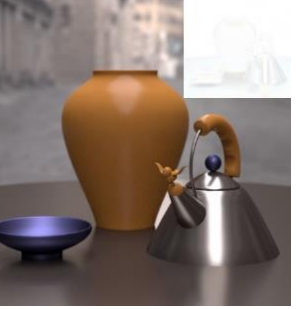

(h) Kurt et al.

$$
(P S N R=30.3722) \quad(P S N R=22.2599) \quad(P S N R=31.7116) \quad(P N S R=32.1210)
$$

Figure 8. A visual comparisons of various well-known BRDF representations on the Princeton scene. While (e) was rendered at 4096 samples/pixel, others were rendered at 262144 samples/pixel. Insets depict differences between the reference image and the rendered images and darker portions in these difference images imply to higher imparity. Below each image we also report PSNR values (higher is better) (images from [16])

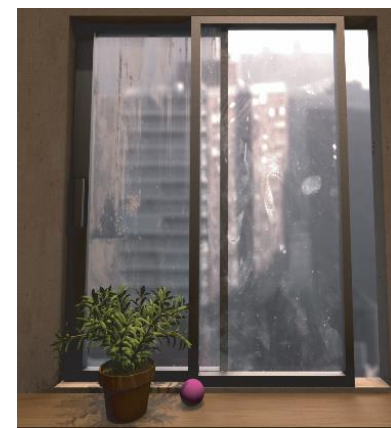

(a) $(6: 00 \mathrm{am})$

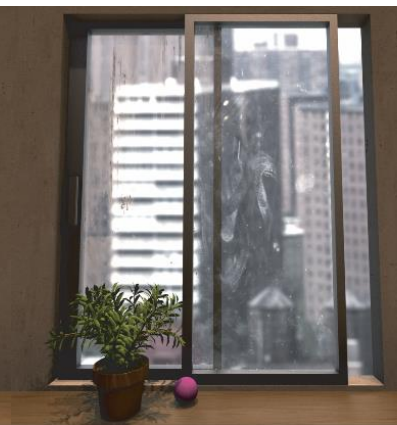

(b) $(14: 00 \mathrm{pm})$

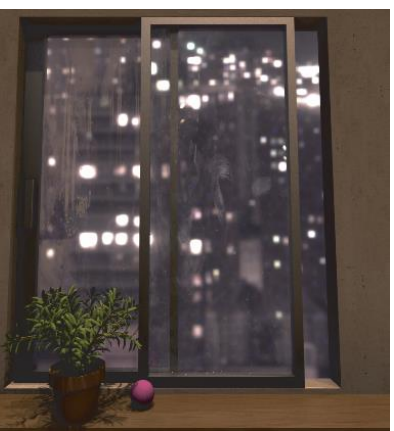

(c) $(1: 00 \mathrm{am})$

Figure 10. A contaminated window is represented by Gu et al.'s [28] BSDF model at different times of a day (images from [28])

also reported to see differences between the BRDF representations. Although, analytical BRDF models can represent a large number of materials, they cannot represent all materials quite well $[26,27]$, e.g., translucent materials.

Walter et al.'s [6] analytical BSDF model is based on microfacet theory and it's for representing rough glass material. In their work, Walter et al. introduced GGX microfacet normal distribution which works extremely well when it's compared to Beckmann distribution for representing rough translucent materials [1]. Walter et al. also proposed importance sampling techniques for their BSDF model, which is essential to Monte Carlo rendering algorithms. The reflection part of Walter et al. BSDF model is similar to Cook-Torrance BRDF model [7]. In this work, we fitted Walter et al. BTDF model to some of the BTDF measurements from BME database [4]. 
Walter et al. BTDF model is formalized as:

$$
\begin{aligned}
& f\left(\omega_{i}, \omega_{o}\right) \\
& =k_{s t} \frac{\left|\omega_{i} \cdot \omega_{h t}\right|\left|\omega_{o} \cdot \omega_{h t}\right|}{\left|\omega_{i} \cdot \omega_{n}\right|\left|\omega_{o} \cdot \omega_{n}\right|} \times \\
& \frac{\eta_{o}^{2}(1-F(\cdot)) G(\cdot) D(\cdot)}{\left(\eta_{i}\left(\omega_{i} \cdot \omega_{h t}\right)+\eta_{o}\left(\omega_{o} \cdot \omega_{h t}\right)\right)^{2}},
\end{aligned}
$$

where $\quad \omega_{h t}=-\left(\eta_{i} \omega_{i}+\eta_{o} \omega_{o}\right) / \| \eta_{i} \omega_{i}+$ $\eta_{o} \omega_{o} \|$ is halfway transmission vector, $\omega_{n}$ is surface normal vector, $F(\cdot)$ is Fresnel term (see Eq. (3)), $G(\cdot)$ is shadowingmasking term, $D(\cdot)$ is microfacet normal distribution function, $k_{s t}$ is specular transmission coefficient, $\eta_{i}$ is index of refraction for the incident side of the surface, $\eta_{o}$ is index of refraction for the transmitted side of the surface. To compute Fresnel term, we use Schlick approximation as $F\left(\omega_{i}, \omega_{h t}, f_{0 t}\right)=f_{0 t}+$ $\left(1-f_{0 t}\right)\left(1-\left(\omega_{i} \cdot \omega_{h t}\right)\right)^{5}$ where $f_{0 t}$ is Fresnel coefficient for the transmitted side of the surface. To compute $G(\cdot)$ term, we use Smith approximation [6] as:

$$
\begin{aligned}
& G\left(\omega_{i}, \omega_{o}, \omega_{h t}, \alpha_{t}\right) \\
& \approx G_{1}\left(\omega_{i}, \omega_{h t}, \alpha_{t}\right) G_{1}\left(\omega_{o}, \omega_{h t}, \alpha_{t}\right) .
\end{aligned}
$$

To compute $G_{1}(\cdot)$ and $D(\cdot)$, we use the following GGX distribution:

$$
\begin{aligned}
& D\left(\omega_{h t}, \alpha_{t}\right) \\
& =\frac{\alpha_{t}^{2} \chi^{+}\left(\omega_{h t} \cdot \omega_{n}\right)}{\pi \cos ^{4} \theta_{h t}\left(\alpha_{t}^{2}+\tan ^{2} \theta_{h t}\right)^{2}}, \\
& G_{1}\left(\omega, \omega_{h t}, \alpha_{t}\right) \\
& =2 \frac{\chi^{+}\left(\frac{\omega \cdot \omega_{h t}}{\omega \cdot \omega_{n}}\right)}{1+\sqrt{1+\alpha_{t}^{2} \tan ^{2} \theta}}
\end{aligned}
$$

where $\chi^{+}(a)$ is the positive function, which equals to one if $a>1$ and zero if $a \leq 0$, and $\alpha_{t}$ is width parameter for the transmitted side of the surface. In this process, we followed Ngan et al.'s [26] fitting procedure. We apply a constrained nonlinear optimization technique, estimate $\alpha_{t}, \eta_{o}, \eta_{i}, f_{0 t}$ terms nonlinearly

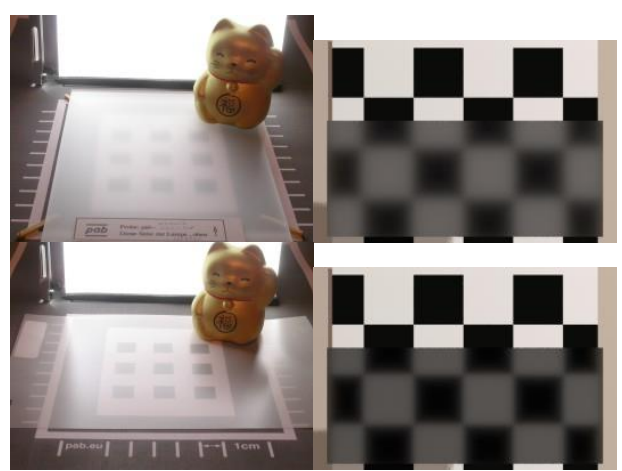

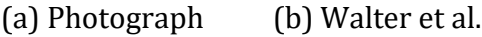

Figure 9. Walter et al.'s analytical BTDF model [6] (b) represents the transmission of isotropic translucent materials (a) visually plausibly. While first row is L02 - 148 material, second row is vk_op10 material from the BME database [4]

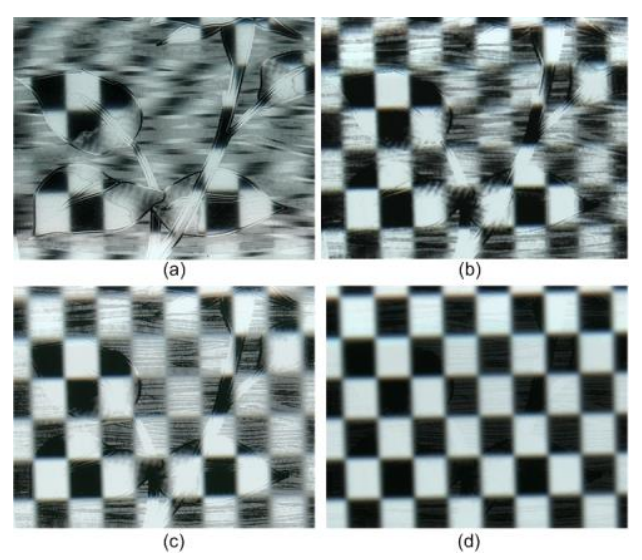

Figure 11. The leaf glass with rough pattern in the front of the checker board is represented by various material models. (a) Real image, (b) Dai et al.'s [18] SVBTDF model, (c) Walter et al.'s [6] BTDF model, (d) $\mathrm{Gu}$ et al.'s [28] dirty glass model (images from [18])

by using a constrained minimization algorithm. $\quad k_{s t}$ term is computed analytically as a subprocedure based on a linear least square optimization. To optimize fitting results for finding a global minimum, we restart the optimization with a different set of initial guesses and we take a set of parameters which leads the minimum $L^{2}$ error. As it can be seen in Figure 9, Walter et al. BTDF model can be used to represent 
transmission of isotropic rough glass materials.

$\mathrm{Gu}$ et al. [28] extended Hanrahan and Krueger's [31] multilayered surface model to represent BSDFs of transparent materials, which include contaminants, such as dust, dirt and lipids. Gu et al. [28] tried to represent a thin transparent slab with a contamination layer. Therefore, $\mathrm{Gu}$ et al.'s [28] BSDF model includes the following components: mirror reflection at the surface of the contaminant layer (BRDF), mirror reflection at the surface of the transparent layer (BRDF), single scattering of the contaminant layer (BRDF), single scattering of the light reflected from the transparent layer (BRDF), transmission of the incident light at both layers (BTDF) and scattering into the transparent layer (BTDF). Gu et al.'s BSDF model has 4 parameters, namely, the optical thickness of the contaminant layer, which is parameterized by the surface location (see Eq. (1)), the mean cosine of the scattering angle (see Eq. (2)), the albedo of the contaminant layer, the refractive index of the contaminant layer. As it can be seen in Figure 10, Gu et al.'s BSDF model can be used to represent transparent surfaces that include contamination. However, Gu et al.'s BSDF model cannot be used to represent spatial variation of blurring and anisotropy, which can be seen in Figure 11.

Dai et al. [18] proposed a microfacetbased Spatially Varying Bidirectional Transmission Distribution Function (SVBTDF) model for representing thin, transparent materials. Their proposed SVBTDF model is called as the dual microfacet model, since Dai et al. assumed that thin, transparent materials are composed of two independent rough surfaces with two different refractive indexes. Dai et al. [18] tabulated the Microfacet Normal Distribution Function (NDF) as a function of surface location and they used this spatially varying NDF for both surfaces. In the final SVBTDF model, Dai et al. combined approximations of these two surfaces by using linear interpolation in logarithm space. As it can be seen in Figure 11, Dai et al.'s SVBTDF model can be used for representing anisotropic transparent materials. However, Dai et al.'s SVBTDF model is not suitable for representing reflection part of translucent materials.

Jakob et al. [32] presented a general framework for rendering isotropic BSDFs of layered materials. This framework allows layered structures. It can be used to render BSDFs efficiently and accurately. This framework includes a precomputation step that expands measured BRDFs, microfacet models for transmission and reflection, media with Henyey-Greenstein and von Mises-Fisher phase functions into a Fourier basis. Fourier basis allows arbitrary composition and efficient importance sampling. In the precomputation process, the plane-parallel multiple scattering problem is solved by using adding/doubling and adding equations. The resulting BSDF is tabulated and stored to be used for rendering at the end of this process. Jakob et al. also used a multiple scattering term for microfacet BSDFs that solves energy loss issue of these BSDF models. A complete opensource implementation of this framework can be found as a part of Mitsuba renderer [33].

Papas et al. [1] introduced an analytical BSDF model for representing several types of paper material. Papas et al.'s physically-based BSDF representation includes absorption and scattering parameters and accounts for single scattering, multiple scattering, surface reflection and surface transmission. For the surface reflection, Papas et al. used the BRDF component introduced by Walter et al. [6]. Papas et al. used the GGX 

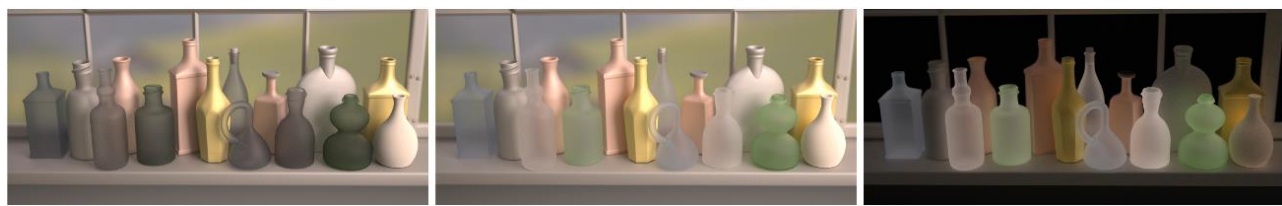

Figure 12. Left: a microsurface single scattering render, which is computed by a traditional BSDF model. Middle: a microsurface single and multiple scattering render, which is computed by Heitz et al.'s [19] BSDF model. Right: $1 \times$ difference image between left and middle images. In this scene, Heitz et al.'s BSDF model introduces $87 \%$ render time overhead, but it provides more accurate and more energy conserving representations (images from [19])

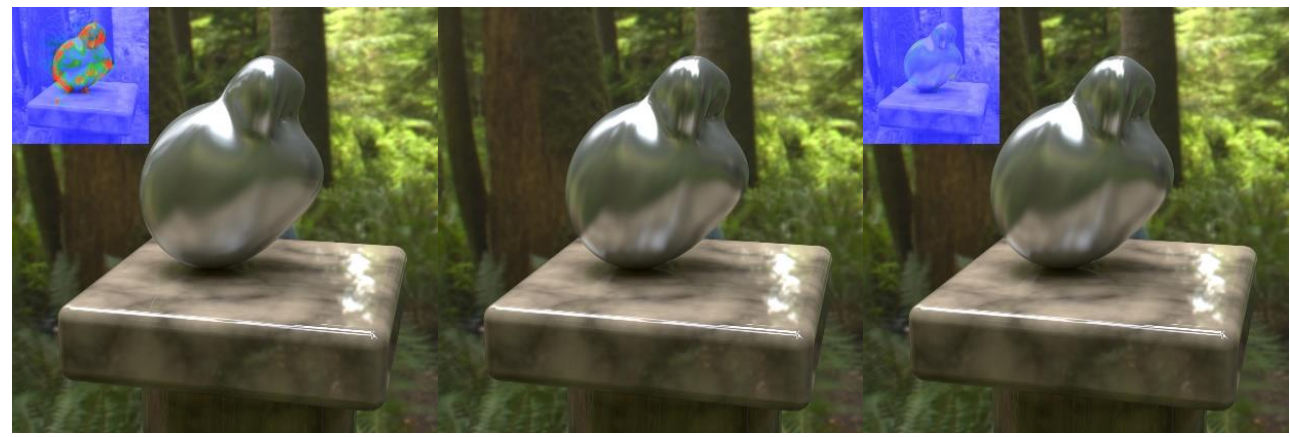

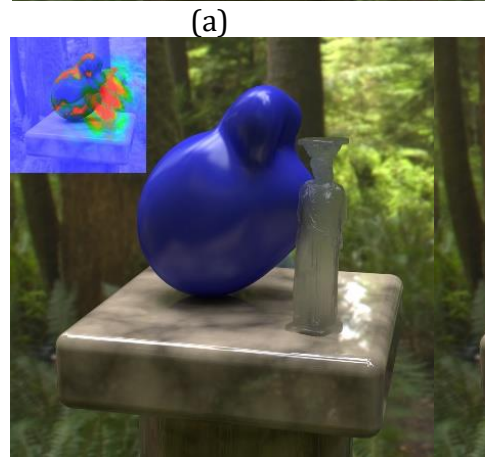

(d) (b)

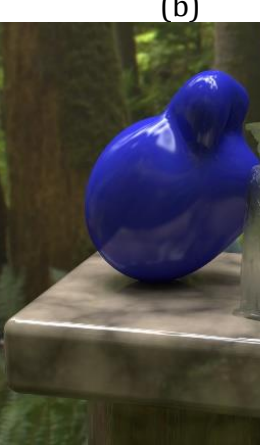

(e) (c)

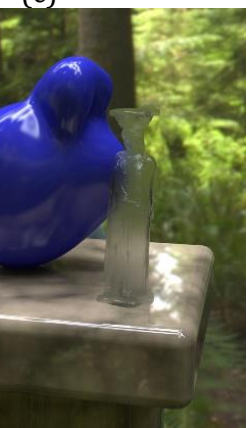

(f)

Figure 13. (a),(d) Matrix-based representation. (b), (e) Reference image. (c), (f) Tensor tree representation. Insets show color-coded differences between reference and rendered images [34] (images from [35])

distribution for representing rough surfaces, such as matte paper and they used the Beckmann distribution for representing smooth surfaces, such as luster and glossy papers (see Figure 1). Since Papas et al. use single and multiple scattering terms in their BSDF model, their BSDF model can be considered as a method for converting a multi-layer Bidirectional Scattering Surface Reflectance Distribution Function (BSSRDF) model $[5,36]$ into a BSDF. All of these terms in Papas et al.'s BSDF model allows a plausible representation of paper materials, which can be seen in Figure 1. However, both Jakob et al.'s general framework and Papas et al. BSDF model are not suitable for representing custom-designed translucent materials that have unusual scattering properties and/or highly anisotropic structures, such as daylight redirecting films.

Heitz et al. [19] proposed an analytical microfacet-based BSDF model, which accounts for microsurface single and multiple scattering terms. Heitz et al.'s BSDF model is the first analytical 
microfacet-based BSDF model that incorporates the multiple scattering component. Both single and multiple scattering terms are represented by Smith microsurface model. Heitz et al. formulated scattering from microfacets as a volumetric scattering process using a new variant of microflake theory. According to that theory, Heitz et al. derived free-path distributions and phase functions for the volume that corresponds to the Smith microsurface. Heitz et al. also introduced a randomwalk approach to microsurface scattering based on the volumetric free paths and phase functions. The random-walk approach makes heavy use of the distribution of visible normals (VNDF) importance sampling technique, which was proposed by Heitz and d'Eon [37]. Heitz et al.'s BSDF model can be considered as the expectation of all of the paths that can be traced on the microsurface. Heitz et al.'s BSDF model is energy conserving, reciprocal and able to represent anisotropic translucent materials. Heitz al.'s BSDF model also has an efficient importance sampling procedure which is based on the random-walk approach. As it can be seen in Figure 12, Heitz et al.'s BSDF model can be used to represent translucent materials more accurately than classical BSDF models that only include single scattering term, for the sake of introducing additional computational overhead. While Jakob et al.'s [32] BSDF framework handles the multiple scattering between different layers, Heitz et al.'s BSDF model focuses on the multiple scattering inside one layer. Therefore, both works can be combined to represent multilayered translucent materials accurately.

Lawrence et al.'s [20] data-driven BRDF representation uses a Non-negative Matrix Factorization (NMF)-based algorithm and it's also suitable for efficient BRDF importance sampling.
Öztürk et al.'s [21] BRDF model is based on Rusinkiewicz coordinate system [25] and uses Copula distributions for representing measured reflectance data. Bilgili et al.'s [22] factored BRDF representation uses a Tucker-based factorization algorithm to compactly represent measured BRDF data and it allows to an efficient BRDF importance sampling. Pacanowski et al. [23] uses rational functions to compactly represent measured reflectance data. Pacanowski et al.'s [23] data-driven BRDF representation is based on Rusinkiewicz coordinate system [25] as this coordinate system helps to represent specular highlights more accurately. However, none of these data-driven BRDF representations can represent noisy, sparse and irregular measurements. Therefore, noisy, sparse and irregular measurements need to be preprocessed before they can be represented with a data-driven based representation. Additionally, these data-driven BRDF representations cannot be used for modeling transmission measurements.

\section{Results and Suggestions}

We found several key issues as a result of our investigation in BSDF literature. In this section, we'll discuss these key issues. Then, we'll suggest some research topics, which could be important contributions to BSDF literature.

One of the key issues is filling noisy, sparse and irregular measurements, as many data-driven based BSDF representations, such as Matrix-based BSDF representation and Tensor tree BSDF representation [35], require noisefree, continuous and regular BSDF measurements. Recently, Ward et al. [38] proposed an interpolation technique for filling a sparse set of incident angle BSDF measurements. The proposed interpolation technique is based on a Lagrangian mass-transport solution [39] and it fits a set of radial basis functions to 
each measured distribution, which allows to interpolate between sparse incident directions. The proposed interpolation technique is especially suited for anisotropic BSDFs, because anisotropic BSDF measurements generally include many holes and noise. As it can be seen in Figure 14, Ward et al.'s [38] interpolation technique is better than a naive linear interpolation. For efficient rendering and simulation, interpolated data can be converted to a standard BSDF representation, such as Tensor tree representation, and it can be used in a model free framework [35]. However, both Ward et al.'s data-driven BSDF framework [35] and interpolation technique [38] must be properly validated and compared with existing techniques in order to become common standards in computer graphics community.

Another key issue is creating a general framework for sharing and rendering measured BSDFs. Accordingly, Ward et al. [35] proposed an XML representation and an Open Source C library to support BSDFs in rendering applications. The proposed library allows for the efficient representation, query and Monte Carlo sampling of real-world BSDFs in a modelfree framework. The proposed library includes two data-driven based BSDF representations: Matrix-based BSDF representation and Tensor tree BSDF representation. Matrix based BSDF representation has advantages for certain matrix operations. On the other hand, Tensor tree BSDF representation has an adaptive density which helps to represent highly peaked data more accurately. As it can be seen in Figure 13, Tensor tree BSDF representation provides a more accurate representation of measured BSDF data than Matrixbased BSDF representation. Furthermore, Ward et al.'s [35] proposed library helps to handle advanced schemes such as Complex Fenestration
Systems (CFSs) which have been designed to convey daylight in specialized ways, such as prismatic glazings, holographic films, daylight redirecting films and specular louvers. Simulating CFSs correctly is especially important to modern building designers. As it can be seen in Figure 15, CFSs can be simulated more correctly when the data-driven BSDF representation is used as a proxy for detailed geometry. In this setting, the geometry is used for direct views and shadow testing and the datadriven BSDF representation is used for characterizing light reflected and transmitted by the CFS.

Although, a popular database for BRDF measurements is presented by Matusik et al. [2], there is a need of a similar database for BSDF measurements, which can be used for validation, comparison and simulation purposes. Some BSDF representations [19] are only validated on simulated data in the absence of such a well-known BSDF database. A similar situation is valid for existing data fitting and visualization tools. There are a few available data fitting and visualization tools for BRDFs, such as BRDFLab [40], BRDF Explorer [41], and ALTA Library [42]. BRDFLab [40] allows to display analytical, measured and simulated BRDFs, fit any measured BRDF to a combination of analytical models, realtime rendering with point light or environment map light sources. BRDF Explorer [41] allows to compare analytical BRDF models with measured BRDF data, real-time editing of parameters, and visualizations in Rusinkiewicz coordinate system [25]. ALTA library [42] allows rational [23] fitting of measured BRDFs, non-linear fitting of measured BRDFs to an analytical BRDF model, and statistical analysis of BRDF data. We think that such similar data fitting and visualization tools are needed for BSDFs as well. There are only some implementations for data 


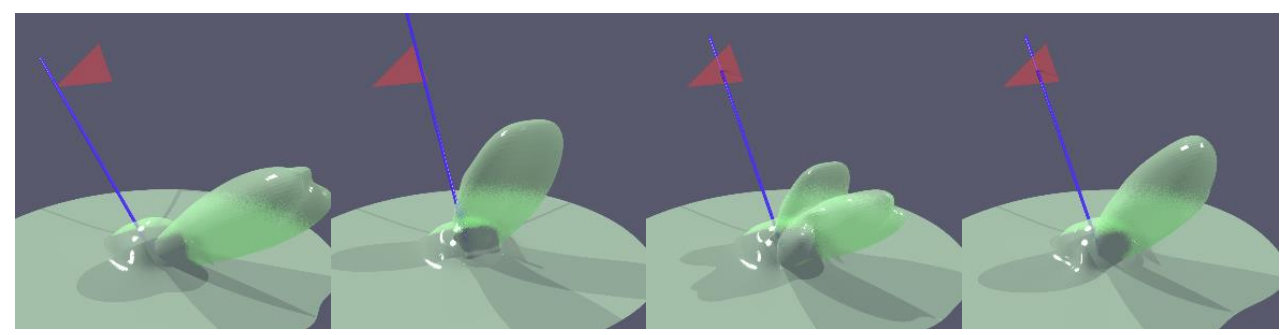

(a)

(b)

(c)

(d)

Figure 14. (a) Measured reflectance distribution at one incident direction. (b) Distribution at another incidence direction. (c) Linear interpolation of three distributions. (d) Lagrangian mass transport based interpolation (images from [38])

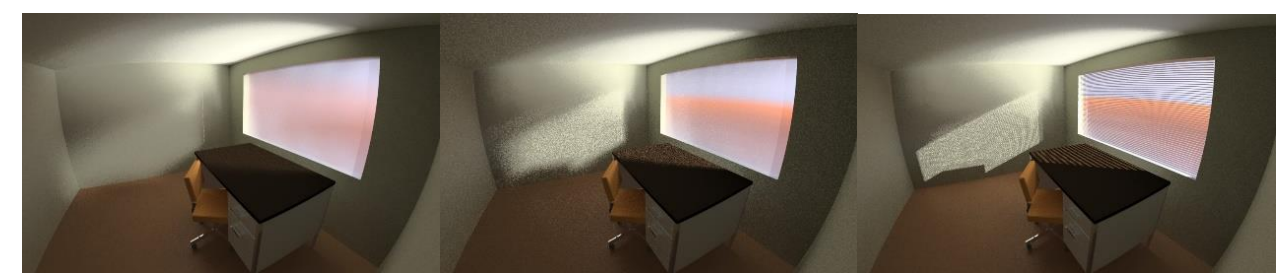

(a) (c)

Figure 15. (a) A venetian blind system was rendered using a $145 \times 145$ Klems matrix representation. (b) was rendered using a tensor tree representation with $3 \times$ the resolution of the Klems matrix data. (c) was rendered using a BSDF surface as a proxy for detailed blinds geometry. We can now see all details and the striped shadows (images from [35])

fitting and visualization of BSDFs in RADIANCE [43]. RADIANCE [43] allows to interpolate measured BSDF data, represent measured BSDF data with data-driven BSDF representations [35], and compare fitting results with BSDF measurements. We believe that much more data fitting, editing and visualization tools are still needed for BSDF models and measurements.

\section{Conclusions}

In this paper, an analysis of the state-ofthe art BSDF measurements and representations has been presented. The strengths and the weaknesses of the investigated BSDF measurements and representations have been discussed. We have showed that the most of the state-of-the-art BSDF models do not suggest a general solution for any surface class, from glasses to metals, isotropic to anisotropic materials, and daylight redirecting films. Furthermore, we have summarized grand challenges and key issues in BSDF measurement and representation. We hope that this short outline of the key issues encourages researchers to focus on these issues, and helps to advance BSDF measurement and representation.

Indeed, there is a need for a huge database that includes variety of BSDF measurements. We think that both researchers and designers will use such a database for comparison, validation and simulation purposes. In the future, we're planning to help this process by measuring various translucent materials and providing them with a suitable BSDF library. In BSDF representation side, there is a need for an accurate interpolation technique and an efficient BSDF representation. Extrapolating BSDF data at grazing angles and modeling backlit appearance are other big challenges and they should be carefully handled. In the future, we're also planning to investigate an accurate BSDF representation that handles these challenges quite well. 


\section{Acknowledgments}

This work was supported by the Scientific and Technical Research Council of Turkey (Project No: 115E203), the Scientific Research Projects Directorate of Ege University (Project No: 2015/BİL/043). Author would like to thank Marios Papas for sharing the scenes in Figure 1 and Figure 7; Özkan Anıl Töral and Tanaboon Tongbuasirilai for proofreading the paper; and the anonymous reviewers for their constructive comments. Author would also like to thank all the co-authors of the papers discussed here, as well as other collaborators that have helped when preparing this work.

\section{References}

[1] Papas, M., de Mesa, K., Jensen, H.W. 2014. A Physically-Based BSDF for Modeling the Appearance of Paper, Computer Graphics Forum, Vol. 33, No. 4, pp. 133-142. (Proc. Eurographics Symp. Rendering '14).

[2] Matusik, W., Pfister, H., Brand, M., McMillan, L. 2003. A data-driven reflectance model, ACM Transactions on Graphics, Vol. 22, No. 3, pp. 759-769. (Proc. SIGGRAPH '03).

[3] Ghosh, A., Achutha, S., Heidrich, W., O'Toole, M. 2007. BRDF acquisition with basis illumination. International Conference on Computer Vision, 1-8.

[4] Apian-Bennewitz, P. 2014. Building material examples (BME) BRDF and BSDF database. http://www.pab.eu/goniophotometer/demodata/bme/ (Access Date: 15.11.2017).

[5] Nicodemus, F.E., Richmond, J.C., Hsia, J.J., Ginsberg, I.W., Limperis, T. 1977. Geometrical Considerations and Nomenclature for Reflectance. Final Report National Bureau of Standards, Washington, DC. Inst. for Basic Standards, National Bureau of Standards (US).

[6] Walter, B., Marschner, S.R., Li, H., Torrance, K.E. 2007. Microfacet models for refraction through rough surfaces. The 18th Eurographics Conference on Rendering Techniques, ser. EGSR'07, 195-206.

[7] Cook, R.L., Torrance, K.E. 1981. A reflectance model for computer graphics, Computer Graphics, Vol. 15, No. 3, pp. 307-316.

[8] He, X.D., Torrance, K.E., Sillion, F.X., Greenberg, D.P. 1991. A comprehensive physical model for light reflection, Computer Graphics, Vol. 25, No. 4, pp. 175-186. (Proc. SIGGRAPH '91).

[9] Ward, G.J. 1992. Measuring and modeling anisotropic reflection, Computer Graphics, Vol. 26, No. 2, pp. 265-272. (Proc. SIGGRAPH '92).

[10] Lafortune, E.P., Foo, S.-C., Torrance, K.E., Greenberg, D.P. 1997. Nonlinear approximation of reflectance functions. SIGGRAPH '97, 117-126.

[11] Ashikhmin, M., Shirley, P. 2000. An anisotropic Phong BRDF model, Journal of Graphics Tools, Vol. 5, No. 2, pp. 25-32.

[12] Duer, A. 2005. On the Ward model for global illumination. Unpublished material.

[13] Edwards, D., Boulos, S., Johnson, J., Shirley, P., Ashikhmin, M., Stark, M., Wyman, C. 2006. The halfway vector disk for BRDF modeling, ACM Transactions on Graphics, Vol. 25 , No. 1 , pp. 1-18.

[14] Ozturk, A., Kurt, M., Bilgili, A., Gungor, G. 2008. Linear approximation of bidirectional reflectance distribution functions, Computers \& Graphics, Vol. 32, No. 2, pp. 149-158.

[15] Geisler-Moroder, D., Dür, A. 2010. A new ward BRDF model with 
bounded albedo, Computer Graphics Forum, Vol. 29, No. 4, pp. 1391-1398. (Proc. Eurographics Symp. Rendering '10).

[16] Kurt, M., Szirmay-Kalos, L., Krivanek, J. 2010. An anisotropic brdf model for fitting and monte carlo rendering. SIGGRAPH Computer Graphics, Vol. 44, No. 1, pp. 1-15.

[17] Xu, K., Sun, W.-L., Dong, Z., Zhao, D.Y., Wu, R.-D., Hu, S.-M. 2013. Anisotropic spherical Gaussians. ACM Transactions on Graphics, Vol. 32, No. 6, pp. 209:1-209:11. (Proc. SIGGRAPH Asia'13).

[18] Dai, Q., Wang, J., Liu, Y., Snyder, J., Wu, E., Guo, B. 2009. The dualmicrofacet model for capturing thin transparent slabs, Computer Graphics Forum, Vol. 28, No. 7, pp. 1917-1925. (Proc. Pacific Graphics '09).

[19] Heitz, E., Hanika, J., d'Eon, E., Dachsbacher, C. 2016. Multiplescattering microfacet bsdfs with the smith model, ACM Transactions on Graphics, Vol. 35, No. 4, pp. 58:1-58:14. (Proc. SIGGRAPH '16).

[20] Lawrence, J., Rusinkiewicz, S., Ramamoorthi, R. 2004. Efficient BRDF importance sampling using a factored representation, ACM Transactions on Graphics, Vol. 23, No. 3, pp. 496-505. (Proc. SIGGRAPH '04).

[21] Öztürk, A., Kurt, M., Bilgili, A. 2010. A copula-based brdf model, Computer Graphics Forum, Vol. 29, No. 6, pp. 1795-1806.

[22] Bilgili, A., Öztürk, A., Kurt, M. 2011. A general BRDF representation based on tensor decomposition, Computer Graphics Forum, Vol. 30, No. 8, pp. 2427-2439.

[23] Pacanowski, R., Celis, O.S., Schlick, C., Granier, X., Poulin, P., Cuyt, A. 2012. Rational BRDF, IEEE Transactions on Visualization and Computer Graphics, Vol. 18, No. 11, pp. 1824-1835.

[24] Guarnera, D., Guarnera, G., Ghosh, A., Denk, C., Glencross, M. 2016. Brdf representation and acquisition, Computer Graphics Forum, Vol. 35, No. 2, pp. 625-650. (Proc. Eurographics STAR - State of The Art Report '16).

[25] Rusinkiewicz, S.M. 1998. A new change of variables for efficient brdf representation. Eurographics Workshop on Rendering, Springer, 11-22.

[26] Ngan, A., Durand, F., Matusik, W. 2005. Experimental analysis of BRDF models, Eurographics Symposium on Rendering, 117126.

[27] Bagher, M., Soler, M.C., Holzschuch, N. 2012. Accurate fitting of measured reflectances using a Shifted Gamma micro-facet distribution, Computer Graphics Forum, Vol. 31, No. 4, pp. 15091518. (Proc. Eurographics Symp. Rendering '12).

[28] Gu, J., Ramamoorthi, R., Belhumeur, P.N., Nayar, S.K. 2007. Dirty glass: Rendering contamination on transparent surfaces. The Eurographics Symposium on Rendering Techniques, Grenoble, France, Eurographics Association, 159-170.

[29] Schlick, C. 1994. An inexpensive BRDF model for physically-based rendering, Computer Graphics Forum, Vol. 13, No. 3, pp. 233-246.

[30] Richardson, I.E. 2002. Video Codec Design: Developing Image and Video Compression Systems. John Wiley \& Sons, Inc. New York, NY, USA.

[31] Hanrahan, P., Krueger, W. 1993. Reflection from layered surfaces due to subsurface scattering. SIGGRAPH '93, ACM, 165-174.

[32] Jakob, W., d'Eon, E., Jakob, O., Marschner, S. 2014. A comprehensive framework for 
rendering layered materials, ACM Transactions on Graphics, Vol. 33, No. 4, pp. 118:1-118:14. (Proc. SIGGRAPH'14).

[33] Jakob, W. 2010. Mitsuba renderer. http://www.mitsuba-renderer.org (Access Date: 15.11.2017).

[34] Mantiuk, R., Kim, K.J., Rempel, A.G., Heidrich, W. 2011. HDR-VDP-2: A calibrated visual metric for visibility and quality predictions in all luminance conditions, ACM Transactions on Graphics, Vol. 30, No. 4, pp. 40:1-40:14. (Proc. SIGGRAPH '11).

[35] Ward, G., Kurt, M., Bonneel, N. 2012. A practical framework for sharing and rendering real-world bidirectional scattering distribution functions, Lawrence Berkeley National Laboratory, Tech. Rep. LBNL-5954E, http://eetd.lbl.gov/sites/all/files/ publications/lbnl12-tensor-treerepresentation29102012.pdf

[36] Donner, C., Jensen, H.W. 2005. Light diffusion in multi-layered translucent materials, ACM Transactions on Graphics, Vol. 24, No. 3, pp. 1032-1039. (Proc. SIGGRAPH '05).

[37] Heitz, E., d'Eon, E. 2014. Importance sampling microfacetbased bsdfs using the distribution of visible normal, Computer Graphics Forum, Vol. 33, No. 4, pp.
103-112. (Proc. Eurographics Symp. Rendering '14).

[38] Ward, G., Kurt, M., Bonneel, N. 2014. Reducing anisotropic bsdf measurement to common practice. The 2nd Eurographics Workshop on Material Appearance Modeling: Issues and Acquisition, Eurographics Association, 5-8.

[39] Bonneel, N., van de Panne, M., Paris, S., Heidrich, W. 2011. Displacement interpolation using lagrangian mass transport, ACM Transactions on Graphics, Vol. 30, No. 6, pp. 158:1-158:12. (Proc. SIGGRAPH Asia '11).

[40] Fores, A., Pattanaik, S.N., Bosch, C., Pueyo, X. 2009. BRDFLab: A general system for designing BRDFs. CEIG'09, Eurographics, 153-160.

[41] I. Copyright Disney Enterprises. 2012. Brdf explorer. http://www.disneyanimation.com /technology/brdf.html (Access Date: 15.11.2017).

[42] Belcour, L., Courtes, L., Pacanowski, R., et al. 2015. ALTA: A BRDF Analysis http://alta.gforge.inria.fr/ (Access Date: 15.11.2017).

[43] Ward, G.J. 1994. The radiance lighting simulation and rendering system. SIGGRAPH '94, ACM, 459472. 